\section{Acute crises and complications of sickle cell anemia among patients attending a pediatric tertiary unit in Kinshasa, Democratic Republic of Congo}

\author{
Michel Ntetani Aloni, Bertin Tshimanga \\ Kadima, Pépé Mfutu Ekulu, Aléine \\ Nzazi Budiongo, René Makuala Ngiyulu, \\ Jean Lambert Gini-Ehungu \\ Division of Pediatric Hemato-Oncology \\ and Nephrology, Department of \\ Pediatrics, University Hospital of \\ Kinshasa, School of Medicine, \\ University of Kinshasa, Democratic \\ Republic of Congo
}

\begin{abstract}
In the Democratic Republic of Congo, the incidence of sickle cell anemia (SCA) is estimated to affect 30,000 to 40,000 neonates per year. However, there is paucity of data on acute clinical manifestations in sickle cell children. In these circumstances, it is difficult to develop a health care policy for an adequate management of sickle cell patients. This was a seven years' retrospective study of children admitted with acute sickle cell crisis in the Department of Pediatrics in University Hospital of Kinshasa, Kinshasa, the Democratic Republic of Congo. A total of 108 patients were identified as having SCA. There were $56(51 \%)$ girls and 52 (49\%) boys. Median age was 10.5 years (range $1-24$ years). No child was diagnosed by neonatal screening. The median age of diagnosis of sickle cell anemia was 90 months (range: 8-250 months). The median age at the first transfusion was 36 months (range 4-168). In this series, $61(56.5 \%)$ patients were eligible for hydroxyurea. However, this treatment was only performed in $4(6.6 \%)$ of them. Pain episodes, acute anemic crisis and severe infection represent respectively $38.2 \%$, $34.3 \%$ and $21.9 \%$ of events. Altered sensorium and focal deficit were encountered occasionally and represented $3.4 \%$ of acute events. Acute renal manifestations, cholelithiasis and priapism were rarely reported, in this cohort. In Kinshasa, the care of patients suffering from sickle cell anemia is characterized by the delayed diagnosis and low detection of organ complications compared to reports of Western countries. This situation is due to resources deficiencies.
\end{abstract}

\section{Introduction}

In Democratic Republic of Congo (DRC), the prevalence of sickle cell anemia (SCA) is extremely high with 25 to $30 \%$ of sickle cell trait in the general population and SCA affects approximately $1.4 \%$ of newborns. ${ }^{1-3}$ As per estimates, about 40,000 newborns per year are born with homozygous form in this country. Large spectrum of clinical manifestations has been described in other parts of the world. ${ }^{4}$ Though the high incidence and prevalence of SCA and though most patients live in developing countries, there is paucity of data on acute events of hospitalized sickle cell patients from the DRC. Information are necessary for health plan to have the main clinical manifestations and complications of all sickle cell patients followed in the DRC. Timely initiation of antibioprophylaxis, specific vaccine such as conjugate vaccines against both Streptococcus pneumoniae and Haemophilus influenzae type b (Hib), the prompt recognition of sickle cell crisis and their treatment are the only economic viable options. ${ }^{4}$ In this context, knowledge regarding the pattern of acute events in SCA will be useful. In the present study, we retrospectively analyzed clinical characteristics of acute clinical manifestations of Congolese children and young adults suffering from SCA living in a resource-deprived setting and this profile in a tertiary Hospital from Kinshasa. Kinshasa is the largest city in DRC.

\section{Materials and Methods}

\section{Study setting and design}

This study was a retrospective and descriptive study carried on all patients with a diagnosis of sickle cell disease (SCD), who were seen between January 2000 and December 2007 at the Department of Pediatrics of the University Hospital of Kinshasa, Kinshasa, the DRC. The Hospital is a tertiary level teaching hospital and referral center located in Western part of the country. The institution comprises 1000 beds of which 50 in pediatrics. We used hospital-based data from the records because there is no population-based hemoglobinopathies registry in the DRC.

The diagnosis of SCD was determined by cellulose acetate or citrate agar electrophoreses in Laboratory of INRB or Laboratory of Hematology of University Hospital of Kinshasa. All patients were found with SCA (Hb-SS), the only form of SCD that has been documented in DRC. ${ }^{3}$
Correspondence: Michel Ntetani Aloni, Division of Pediatric Hemato-Oncology and Nephrology, Department of Pediatrics, University Hospital of Kinshasa, School of Medicine, University of Kinshasa, PO.BOX 123 Kinshasa XI, the Democratic Republic of Congo.

E-mail: michelaloni2003@yahoo.fr

Key words: Children; acute events: sickle cell anemia; Kinshasa; Democratic Republic of Congo.

Acknowledgements: the authors would like to appreciate all students of Department of Pediatrics for their assistance in collecting data.

Contributions: the authors contributed equally

Conflict of interest: the authors declare no potential conflict of interest.

Received for publication: 29 October 2016. Revision received: 11 February 2017.

Accepted for publication: 17 February 2017.

This work is licensed under a Creative Commons Attribution-NonCommercial 4.0 International License (CC BY-NC 4.0).

CCopyright M.N. Aloni et al., 2017

Licensee PAGEPress, Italy

Hematology Reports 2017; 9:6952

doi:10.4081/hr.2017.6952

\section{Study subjects}

The study included all children who admitted with acute crisis. Medical records were then retrospectively reviewed as recorded in hematology register. Patients who had incomplete or missed basic information were excluded from the study.

Multiple sickle cell crisis occurring in the same patient were considered new-separate episodes, if more than 10 days since the start of the previous crisis had elapsed and clinical improvement had been detected. Thus, the same patient could contribute to multiple episodes of sickle cell crisis in this analysis.

Eligible criteria for hydroxyurea (HU) therapy in our cohort were (i) frequent blood transfusions (more than three per year); (ii) frequent vasoocclusive crises (more than three per year) requiring hospitalization; (iii) at least one of the following neurologic symptoms: stroke, hemiplegia, transient ischemic attacks, seizures, coma or sensory loss; (iv) presence of avascular necrosis of bone; (v) at least one episode of acute chest syndrome. The dose of oral HU is $(20 \mathrm{mg} / \mathrm{kg} /$ day $)$ and was given in eligible children.

Malaria was defined as the presence of 
Plasmodium using both blood films (thin or thick blood smears). Study participants who were positive for malaria were treated following the national guidelines. All pediatricians would have had access to these guidelines at the time of the study. Children diagnosed with severe malaria were treated with intravenous quinine $(20 \mathrm{mg} / \mathrm{kg}$, followed by $10 \mathrm{mg} / \mathrm{kg}$ tid for 7 days).

The species of pathogens were determined by blood or urine cultures in the Department of Microbiology at University Hospital of Kinshasa.

A severe painful was defined as pain in the extremities, abdomen, chest, back, or head for which there was no other explanation (osteomyelitis, appendicitis) and required hospitalization for more than 48 hours. Hyperhemolysis crisis was diagnosed in the presence of exacerbation of anemia without identifiable causes of red cell destruction. Acute splenic sequestration was diagnosed in the presence of a tender, rapidly enlarging spleen accompanied by a decreased hemoglobin concentration from baseline by at least $2 \mathrm{~g} / \mathrm{dL}$. Acute hepatic sequestration was diagnosed in the presence of a tender, progressive enlarging hepatomegaly with accentuated anemia below baseline hyperbilirubinemia and increased count of reticulocytes. Acute chest syndrome was defined as an acute illness characterized by fever respiratory symptoms and/or fever associated with pulmonary infiltrate on chest X-ray. All patients with suspected ACS were treated as severe pneumonia. Priapism was defined as a sustained penile erection in the absence of sexual activity or desire. Cholelithiasis was diagnosed in the presence of gallstones at ultrasonography of the gallbladder when an echodense images casting an acoustic shadows were seen.

\section{Ethical approval}

The study protocol was approved by the institutional authorities and the local ethical committee of the Department of Pediatrics of the University Hospital of Kinshasa. As the study was retrospective, the authorities waived that the research could be done based on record review without contacting the patients. Support letter was obtained from the medical director office of the Department of Pediatrics for retrieving retrospective data from the database and records. All the information was kept confidential, and no individual identifiers were collected.

\section{Statistical analysis}

Information obtained was entered into Microsoft excel file and analysis by EpiInfo version 6. Descriptive data are expressed as numbers, percentages. Data are represented as means $\pm \mathrm{SD}$ when the distribution was normal and median with range when the distribution was not normal (explain) and were calculated for continuous variables. Normal distribution was defined as an arrangement of a data set in which most values cluster in the middle of the range and the rest taper off symmetrically toward either extreme.

\section{Results}

A total of 142 patients were identified as having SCA. Of these, complete data were available on $108(76.1 \%)$ cases. There were $56(51 \%)$ girls and $52(49 \%)$ boys treated in the study period with a female to male ratio of 1.1:1. Overall median age of the study group was 10.5 years (range 1-24 years).

Table 1 shows previous medical history in all patients at admission. The median age of diagnosis of SCA was 90 months (range; $8-250$ months). The median of number of transfusion was 3 (range $0-8$ ). The median age at the first transfusion was 36 months (range 4-168).

Pallor, a systolic murmur heard loudest over the left sternal border and apex and grade 1 to $3 / 6$ intensity and jaundice were the common symptoms at presentation (89.8\%, 88.0\% and $77.8 \%$ respectively). Splenomegaly was present in approximately $25 \%$ of children. Others clinical findings were showed in Table 2.

We classified 237 episodes which occurred in 108 patients (2.2 episodes per patient). Among these 237 episodes, 89 were classified as pain events, 80 as acute anemic crises and 51 as episodes of severe infections. Others acute events were presented in Table 2.

Pain crisis were the first cause of hospitalization and represented $37.6 \%$ of acute events. Bone pain was the most common localization and represented $57.3 \%(n=89)$ episodes. Hand foot syndrome was present in $25 \%$ of patients with pain episodes. Details of pain crisis are presented in Table 2 . Acute chest syndrome was strongly suspected in 6 children. All patients with suspected ACS were treated as severe pneumonia. Acute anemic crisis was the second cause of admission with a frequency of $36.0 \%(n=237)$. Among the 80 acute hematologic crises, hyperhemolysis crisis was the most frequent $(91.3 \%)$. The presumed triggers of hyperhemolytic crises were malaria in $78.8 \%(n=80)$ episodes, bacterial sepsis in $11.3 \%$ episodes $(\mathrm{n}=80)$ and $10 \%$ $(\mathrm{n}=80)$ with unknown cause. Six on 80

Table 1. Distribution of medical history of 108 children at admission.

\begin{tabular}{lcc} 
& Frequency, $\mathbf{n}(\mathbf{n}=\mathbf{1 0 8})$ & $\%$ \\
Expanded Vaccination Program & 96 & 88.9 \\
Folic acid & 86 & 90.0 \\
\hline Sickle cell in family & 60 & 55.6 \\
Blood transfusion & 45 & 41.7 \\
\hline Previous hand foot syndrome & 21 & 19.4 \\
Regular antibioprophylaxis & 14 & 13.0 \\
\hline Previous severe infection & 10 & 9.3 \\
Immunization against S. pneumoniae & 4 & 3.7 \\
\hline Immunization against H. influenzae & 0 & 0 \\
Immunization against Salmonella & 0 & 0 \\
\hline Neonatal screening & 0 & 0 \\
\hline
\end{tabular}

Table 2. Presenting features and physical findings of 108 children with sickle cell anemia.

\begin{tabular}{lcc} 
Symptoms and signs & Frequency, $\mathbf{n}(\mathbf{n}=\mathbf{1 0 8 )}$ & $\%$ \\
Pallor & 97 & 89.8 \\
Systolic head loudest & 95 & 88.0 \\
\hline Jaundice & 84 & 77.8 \\
Splenomegaly & 27 & 25.0 \\
\hline Hepatomegaly & 21 & 19.4 \\
Abdominal bloating & 18 & 16.7 \\
\hline Leg ulcer & 2 & 1.9 \\
\hline
\end{tabular}


$(7.5 \%)$ episodes of hematological crisis were due to splenic sequestration. Two of these children underwent splenectomy due to recurrent episode of splenic sequestration. Aplastic crisis was suspected in one patient but no positive culture of parvovirus B19 was found. Red blood cells transfusions were frequently administered. In this series, $87.5 \%(n=80)$ of patients received blood transfusion when anemia endangers life.

Forty-one episodes of infection were found in this study, of these 19 episodes were pneumonia and 18 episodes of septicemia. Infections represented $21.5 \%$ of all acute events sent in our patients. No positive culture of cerebrospinal fluid was found. All patients had received antibiotherapy before the diagnosis of meningitis but the CSF findings were compatible with bacterial aspect. Details of other severe infections are presented in Table 3.

Febrile malaria episodes were found in 35 patients. Among them, 26 cases were associated with severe anemia $(\mathrm{Hb}<6 \mathrm{~g} / \mathrm{dL})$. No case of cerebral malaria and blackwater fever were found in this series. Febrile urinary tract infections were documented in 14 patients. Klebsiella, E.coli and Proteus were present in respectively, 8, 5 and 1 case.

Stroke was encountered occasionally and was found in $8(7.4 \%)$ documented cases $(n=108)$ and represented $3.4 \%$ of acute events. The scanners for 5 patients had revealed ischemic occlusion in 4 cases and association of previous ischemic sequelae and acute hemorrhage in one case. No MRI was practiced for technical availability reasons. No transfusionnal program was applied to prevent recurrent stroke because of insufficient conditions for long-term transfusion. Others acute events are showed in Table 3.

In this group study, 61 patients were eligible for hydroxyurea. However, this treatment was only performed in four $(6.6 \%)$ patients.

\section{Discussion}

Despite the prevalence and incidence of SCA in DRC, our study is the first attempt to describe the acute clinical manifestations in the population suffering from SCA in our midst. The total number of children in this study indicates that only a tiny fraction of sickle cell Congolese patients in crises benefited from treatment in our tertiary referral center. It is common for some children suffering from sickle cell crises to be taken to prayer houses or traditional healers instead of hospital. This situation is due to cultural beliefs, stigma, and ignorance.

In our series, the diagnosis and routinely management of SCA in Kinshasa is delayed. This is in consistent with related studies from DRC and from developing countries. $^{6-10}$ This situation is due to the absence of a systematic newborn screening program for SCA and the absence of effective integration of SCA in primary health care in the Congolese health care system.

Tardive diagnosis of SCA in Kinshasa calls for implementation of neonatal screening in public health institutions. In addition, in sub-Saharan Africa, more than $90 \%$ of children with SCA die early before the diagnosis can be made. Bacterial infections are the major cause of morbidity and mortality in children with SCA in this part of the world. ${ }^{11}$ It was also observed in our series that immunization against Streptococcus pneumoniae $(3.4 \%)$ and no case against Haemophilus influenzae, Salmonella sp. and hepatitis B virus are insufficiently applied. The major reason for this is resources deficiency. Most of the parents of our patients are poor and because a health care insurance facility is not available in DRC. Thus, these recommended vaccinations not covered by the WHO Expanded Vaccination Program are not affordable to many of the families. An additional factor is the unavailability and high cost of this recommended vaccination in our environment. Similar observations were reported in other studies in developing countries. ${ }^{7,12}$

Clinical findings reported in this study were found to be similar to those described elsewhere. ${ }^{6,7,13-15}$

Sickle cell crisis found in our study were as classical reported in the literature. $6,7,13-15$ In our series, pain crisis was the most frequent cause of admission and comprised $37.6 \%$ of cases. These findings are consistent with previous reports from African centers and other parts of the world. ${ }^{13,14,16}$ Problems which can precipitate vasoocclusive crisis were poorest health condition such as living in limited access to drinking water, to information and limited access to health service as reported in DRC. ${ }^{17}$

The frequency of ACS in our study was lower and similar to those reported by others African authors. ${ }^{7,8,15}$ However, this prevalence rate increases in Western countries with acute chest syndrome ranging to $15.2 \%$ to $24 \%$ of patients. ${ }^{13,16,18}$ Our low prevalence is probably due to underestimation of this sickle cell complications associated with pneumonia episodes in our area. Acute anemic crisis was the second leading cause of hospitalization in $33.8 \%$. The fre-

Table 3. Spectrum of 237 sickle cell acute events in the 108 study patients.

\begin{tabular}{lcc} 
Acute sickle cell crisis & N. & $\%$ \\
Vasoocclusives crisis & 89 & 37.6 \\
Bone pain & 51 & 21.5 \\
Hand foot syndrome & 22 & 9.3 \\
Abdominal pain & 9 & 3.8 \\
Acute chest syndrome & 6 & 2.5 \\
Acute hematologic crisis & 80 & 33.8 \\
Hyperhemolysis & 73 & 30.8 \\
Sequestration of spleen & 6 & 2.5 \\
Aplastic crisis & 1 & 0.4 \\
\hline Severe bacterial infection & 51 & 21.5 \\
Pneumonia & 19 & 8.0 \\
Septicemia & 18 & 7.6 \\
Osteomyelitis & 9 & 3.8 \\
Meningitis & 4 & 1.7 \\
Typhoid fever & 1 & 0.4 \\
Altered sensorium and focal deficit & 8 & 3.4 \\
Ischemic occlusion & 4 & 1.7 \\
Hemorrhage & 1 & 0.4 \\
Unknown & 3 & 1.3 \\
\hline Acute renal manifestations & 3 & 1.3 \\
Hematuria & 1 & 0.4 \\
Acute renal failure & 1 & 0.4 \\
Nephrotic syndrome & 1 & 0.4 \\
Others & 6 & 2.5 \\
Cholelithiasis & 3 & 1.3 \\
Priapism & 2 & 0.8 \\
Acute leukemia & 1 & 0.4 \\
\hline Total & 237 & 100 \\
\hline
\end{tabular}


quency of acute anemic due to hyperhemolysis observed in this study is in consistent with related studies from previous studies. $7,8,15,19,20$

The frequency of acute spleen sequestration observed in our series was lower in comparison to the Western countries. ${ }^{21}$ Probably, this complication is under-diagnosed by health professionals and a great number of unknown sickle cell patients die before reaching a specialized center for SCA in our setting.

In our study, severe infections are the third leading cause of hospitalization and were found in $21.5 \%$. In this respect, our findings are similar to previous reports in Africa. ${ }^{15,20}$ In contrast, infections are major cause of hospitalization in Western countries. ${ }^{13,18,21}$ This situation is due to the absence of a systematic newborn screening program for SCA in the Congolese health care system. Neonatal screening is not recommended in the health care policy. This situation suggests that many SCA patients could have died from severe infectious complications before the establishment of diagnosis of SCA as reported in the African literature. ${ }^{11,22}$ A recent report in DRC shows that approximately $8.0 \%$ of children with severe infections carried unknown SCA mutations. ${ }^{23}$

Malaria was an important cause of morbidity in this study. This is not surprising as severe malaria has been reported to be a leading cause of pain crisis, high rate of transfusion, hospitalization and mortality in sub-Saharan Africa. ${ }^{11,19,24}$ In the DRC, there is no recommendation of life -long malaria prophylaxis in people with SCA. Previous studies have showed that malaria chemoprophylaxis reduces sickle cell crisis, number of admission and rate of blood transfusion in areas where malaria is endemic. ${ }^{25}$ In this series, no case of cerebral malaria was found. Our observation was similar to those reported in Senegalese cohort. ${ }^{26}$ It has been postulated that the development of Plasmodium falciparum is partially inhibited by the $\mathrm{Hb}$ SS red cells. More recently, this theory has been challenged. Molecular mechanisms may be inhibiting in the etiology of cerebral malaria as it occurs in the general population and confers to patients with SCA protection against cerebral malaria. ${ }^{27}$

Frequencies of stroke, cholelithiasis, priapism, acute chest syndrome and renal disorders observed in the present study are low and similar to those reported in developing countries. ${ }^{7,8,15,20}$ This low frequency is probably due to the deficit of medical devices in this health institution for detection of this sickle cell complications during follow-up of the patients.
Epistaxis was a rare event in this study. Similar observations were previously reported in the African literature. ${ }^{28}$ Our prevalence rate is different to previous study in south of DRC where the prevalence rate was $39.5 \%$ and $52 \%$ in two different series of sickle cell patients. ${ }^{29}$

Hydroxyurea was not given regularly according to eligible criteria in SCA in Kinshasa. The major reason for this is lack of resources. Most of the parents are poor and do not have free health care facility is not available in health politics system in DRC. ${ }^{17}$ An additional factor is the unavailability and high cost of hydroxyurea in Kinshasa.

\section{Conclusions}

In the DRC, SCA is characterized by delayed diagnosis and This report pointing out the problem encountered in the specific and early diagnosis of SCA complications in limited resource-settings.

\section{References}

1. World Health Organization. Updates estimates of frequency of the haemoglobin disorders in each country. Available from: www.who.ch/programme/ncd/ hgm/haemogl.htm. Accessed: 26 January 2010.

2. Agasa B, Bosunga K, Opara A, et al. Prevalence of sickle cell disease in a northeastern region of the Democratic Republic of Congo: what impact on transfusion policy? Transfus Med 2010;20:62-5.

3. Tshilolo L, Aissi LM, Lukusa D, et al. Neonatal screening for sickle cell anemia in the Democratic Republic of Congo: experience from a pioneer project on 31.204 newborns. J Clin Pathol 2009;62:35-8.

4. Kavanagh PL, Sprinz PG, Vinci SR, et al. Management of children with sickle cell disease: a comprehensive review of the literature. Pediatrics 2011;128: e1552-74.

5. WHO. World Malaria Report. Geneva: World Health Organization; 2011. Available from: http://www.who.int/ malaria/world_malaria_report_2011/en/

6. Aloni MN, Nkee L. Challenge of managing sickle cell disease in a pediatric population living in Kinshasa, Democratic Republic of Congo: a sickle cell center experience. Hemoglobin 2014;38:196-200.

7. Diagne I, Diagne-Gueye ND, Signate-
Sy H, et al. Management of children with sickle cell disease in Africa: experience in a cohort of children at the Royal Albert Hospital in Dakar. Med Trop (Mars) 2003;63:513-20.

8. Ayéroué J, Kafando E, Kam L, et al. Hemoglobin sickle cell disease: experience of the Yalgado Ouedraogo University Hospital of Ouagadougou, Burkina Faso. Arch Pediatr 2009;16: 316-21.

9. Zanette AM, Gonçalves Mde S, Bahia $\mathrm{RC}$, et al. Sickle cell anemia: delayed diagnosis in Bahia, Brazil--a largely Afro-descendant population. Ethn Dis 2011;21:243-7.

10. Brown BJ, Akinkunmi BF, Fatunde OJ. Age at diagnosis of sickle cell disease in a developing country. Afr J Med Med Sci 2010;39:221-5.

11. Grosse SD, Odame I, Atrash HK, et al. Sickle cell disease in Africa: a neglected cause of early childhood mortality. Am J Prev Med 2011;41:S398-405.

12. Nacoulma EW, Kam L, Gue EE, et al. Vaccination status of children with sickle cell disease in Ouagadougou (Burkina Faso). Sante 2006;16:155-60.

13. Lê PQ, Ferster A, Cotton F, et al. Sickle cell disease from Africa to Belgium, from neonatal screening to clinical management. Med Trop (Mars) 2010; 70:467-70.

14. Sadarangani M, Makani J, Komba AN, et al. An observational study of children with sickle cell disease in Kilifi, Kenya. Br J Haematol 2009; 146:675-82.

15. Babela JR, Nzingoula S, Senga P. Sickle-cell crisis in the child and teenager in Brazzaville, Congo. A retrospective study of 587 cases. Bull Soc Pathol Exot 2005;98:365-70.

16. Gill FM, Sleeper LA, Weiner SJ, et al. Clinical events in the first decade in a cohort of infants with sickle cell disease. Cooperative Study of Sickle Cell Disease. Blood 1995;86:776-83.

17. Wembonyama S, Mpaka S, Tshilolo L. Medicine and health in the Democratic Republic of Congo: from Independence to the Third Republic. Med Trop (Mars) 2007:67:447-57.

18. Neonato MG, Guilloud-Bataille M, Beauvais P, et al. Acute clinical events in 299 homozygous sickle cell patients living in France. French Study Group on Sickle Cell Disease. Eur J Haematol 2000;65:155-64.

19. Tshilolo LM, Mukendi RK, Wembonyama SO. Blood transfusion rate in Congolese patients with sickle cell anemia. Indian J Pediatr 2007;74: $735-8$

20. Thuilliez V, Ditsambou V, Mba JR, et 
al. Current aspects of sickle cell disease in children in Gabon. Arch Pediatr 1996;3:668-74.

21. Tarer V, Etienne-Julan M, Diara JP, et al. Sickle cell anemia in Guadeloupean children: pattern and prevalence of acute clinical events. Eur J Haematol 2006;76:193-9.

22. Williams TN, Uyoga S, Macharia A, et al. Bacteraemia in Kenyan children with sickle-cell anaemia: a retrospective cohort and case-control study. Lancet 2009;374:1364-70.

23. Kondani DA, Gini-Ehungu JL, Bodi JM, et al. Prevalence of sickle cell disease in a pediatric population suffering from severe infections: a Congolese experience. Hemoglobin 2014;38:2259.

24. Ambe JP, Fatunde JO, Sodeinde OO. Associated morbidities in children with sickle-cell anaemia presenting with severe anaemia in a malarious area. Trop Doct 2001;31:26-7.

25. Oniyangi O, Omari AA. Malaria chemoprophylaxis in sickle cell disease. Cochrane Database Syst Rev 2006;4: CD003489.

26. Diagne I, Soares GM, Gueye A, et al. Infections in Senegalese children and adolescents with sickle cell anemia: epidemiological aspects. Dakar Med
2000;45:55-8.

27. Ferreira A, Marguti I, Bechmann I, et al. Sickle hemoglobin confers tolerance to Plasmodium infection. Cell 2011;145: 398-409.

28. Acquaye JK, Omer A, Ganeshaguru K, et al. Non-benign sickle cell anaemia in western Saudi Arabia. Br J Haematol 1985;60:99-108.

29. Tshilolo L, Mukendi R, Girot R. Sickle cell anemia in the south of Zaire. Study of two series of 251 and 340 patients followed-up 1988-1992. Arch Pediatr 1996;3:104-11. 